\title{
FUNDAMENTOS DE PSICOTERAPIA
}

\section{PSYCHOTHERAPY FUNDAMENTALS}

\section{Julio TORALES ${ }^{1}$, José BRÍTEZ CANTERO².}

${ }^{1}$ Profesor Asistente de Psiquiatría, Facultad de Ciencias Médicas, Universidad Nacional de Asunción, San Lorenzo - Paraguay.

${ }^{2}$ Profesor Titular de Psiquiatría, Facultad de Ciencias Médicas, Universidad Nacional de Asunción, San Lorenzo - Paraguay.

Cómo citar este artículo: Torales J, Brítez Cantero J. Fundamentos de Psicoterapia. Medicina Clínica y Social. 2017;1(2):157-183.

\section{RESUMEN}

La psicoterapia es entendida como un procedimiento destinado a aliviar el sufrimiento humano por medio de recursos psicológicos. Se basa en la relación profesional y humana de un paciente con un terapeuta experto, que utiliza determinados procedimientos psicológicos y conocimientos sobre el psiquismo, para promover el cambio psíquico, aliviar un sufrimiento o favorecer el desarrollo de la persona y de sus vínculos. La psicoterapia se ha convertido en un recurso innegable en el campo de la salud: como medio de tratar los trastornos mentales, de aliviar el sufrimiento y/o de lograr cambios conductuales. Además, su campo de acción se ha extendido a enfermedades físicas o disfunciones sociales que están vinculadas a trastornos psicológicos. En este artículo se presentarán los conceptos generales de psicoterapia, sus características comunes, los enfoques psicoterapéuticos predominantes y su eficacia basada en la evidencia. Se expondrán, además, algunas apreciaciones acerca de la psicoterapia en población pediátrica y en adultos mayores.

Palabras clave: Psicoterapia; Eficacia; Salud Mental; Enfoques psicoterapéuticos.

\section{ABSTRACT}

Psychotherapy is understood as a procedure designed to alleviate human suffering through psychological resources. It is based on the professional and human relationship of a patient with an expert therapist, who uses certain psychological procedures and knowledge about the psyche, to promote psychic change, to alleviate suffering or to favor the development of the person and his/her relationships. Psychotherapy has become an undeniable resource in the field of health: as a means of treating mental disorders, of alleviating suffering and / or of achieving behavioral changes. In addition, its field of action has been extended to physical illnesses or social dysfunctions that are linked to psychological disturbances. This article will present the general concepts of psychotherapy, the predominant psychotherapeutic approaches and their evidence-based effectiveness. Some insights about psychotherapy in the pediatric population and in the elderly will also be presented.

Keywords: Psychotherapy; Effectiveness; Mental health; Psychotherapeutic approaches. 


\section{INTRODUCCIÓN}

La psicoterapia es entendida como un procedimiento destinado a aliviar el sufrimiento humano por medio de recursos psicológicos, y en ese sentido es utilizada desde tiempos pretéritos. Su implementación como modalidad terapéutica científica se instauró hace más de cien años a la fecha.

Distintas etapas marcan su desarrollo hasta que Sigmund Freud, con la construcción del psicoanálisis, abrió un camino definitivo de un modo de abordaje del padecimiento mental, que se diferenció del paradigma predominante hasta ese momento, que explicaba el sufrimiento mental como debido a fenómenos de naturaleza biológica (con Kraepelin, padre de la psiquiatría moderna, como máximo exponente). Desde otras áreas del mismo campo, son significativos los aportes de Wundt, Pavlov y Watson, en lo que respecta a la psicología experimental (1-4).

Se han dado numerosas definiciones al término "psicoterapia", algunas tan extensas que tienen el inconveniente de ser poco específicas. Por practicidad, y a los efectos de este artículo, se expone la siguiente $(2,5)$ : "La psicoterapia se basa en la relación profesional y humana de un paciente con un terapeuta experto, que utiliza determinados procedimientos psicológicos y conocimientos sobre el psiquismo, para promover el cambio psíquico, buscando aliviar un sufrimiento o favorecer el desarrollo de la persona y de sus vínculos".

Asimismo, vale la pena destacar la definición aprobada en agosto de 2012 por la Asociación Americana de Psicología, que versa (6-8): "La psicoterapia es la aplicación informada e intencional de métodos clínicos y posturas interpersonales, derivados de principios psicológicos establecidos con el propósito de ayudar a las personas a modificar sus conductas, cogniciones, emociones y otras características personales en la dirección que los participantes consideren deseable".

Existen otras muchas definiciones de psicoterapia, tal como se mencionó más arriba, así como existen múltiples corrientes psicoterapéuticas. Sin embargo, los autores afirman que un punto en común entre todas ellas, que es un requisito para que se lleve a cabo esta práctica, es que el paciente ha de considerarse implicado en el problema presentado, ya sea en el origen, en el mantenimiento o en las posibilidades para solucionarlo (9).

\section{CARACTERÍSTICAS COMUNES}

Los rasgos que caracterizan y son comunes a toda psicoterapia están dados por (10-12):

- La relación paciente-terapeuta, basado en la confianza del primero y la competencia del segundo;

- El método, dirigido a la psiquis por vía de la comunicación y su instrumento, la palabra (o más precisamente el lenguaje, verbal y preverbal);

- El objetivo, de cura (concepto que varía según el marco referencial teórico que se sustente) sin cuyo propósito no cabe hablar de psicoterapia; $y$,

- Una teoría, que explique la técnica y la haga comunicable.

Esto implica que existen diversos modelos psicoterapéuticos según los criterios tenidos en cuenta, a saber: a. El número de personas intervinientes (individuales, vinculares, grupales); 
b. Su extensión (breves, intermedias, prolongadas); y, c. Tipos de recursos empleados (verbales, corporales, con elementos auxiliares, entre otros). En las últimas décadas dos factores de primordial importancia produjeron un profundo impacto no sólo en la concepción sino en la búsqueda de respuestas adecuadas respecto al tratamiento de los trastornos mentales (1): la globalización y las neurociencias.

- La globalización: La globalización que atraviesa a la humanidad en su conjunto, con sus modificaciones tecnológicas, económicas, políticas, sociales y culturales, y que genera nuevas formas de demanda y atención producto de dichas transformaciones.

Se puede afirmar que la globalización se ha expresado por un crecimiento de los trastornos y una mayor complejidad de los mismos, lo que se acompaña de la necesidad de generar respuestas de salud inmediatas, eficaces, asequibles y accesibles.

- Las neurociencias: El desarrollo de las neurociencias y su incidencia en la investigación y producción de psicofármacos, con la aparición de psicotrópicos con mayor eficacia y sus consecuencias e implicancias en la clínica, han impuesto readecuaciones y cambios continuos.

Muchos autores, entre los que se incluye a Kandel, afirman que existe actualmente un nuevo marco de referencia en la psiquiatría, al conceptualizar los cambios psicológicos producidos por la psicoterapia a partir de cambios neuronales y de transmisores cerebrales, es decir que se está en los albores para comprender definitivamente cómo las intervenciones psicoterapéuticas producen su efecto. Toda la evidencia del impacto de la psicoterapia en el cerebro abre nuevas líneas de investigación para mejorar la comprensión sobre la psicopatología y el tratamiento de los trastornos mentales. Estas líneas son: a) Mecanismos de acción de las psicoterapias; b) Interrelación de los mecanismos de acción de los psicofármacos y de la psicoterapia; y, c) Comprensión más clara de la patogénesis en sí misma y la plasticidad de algunos componentes de los mecanismos patogénicos de los desórdenes psiquiátricos mayores (13).

Asimismo, es importante puntualizar que la información creciente que sustenta la base neurobiológica de la psicoterapia permite vislumbrar un nuevo horizonte para la psiquiatría. La interrelación entre mente y cerebro, psicología y biología, farmacoterapia y psicoterapia se sitúa actualmente en la vanguardia de la neurociencia (14).

Los investigadores están reuniendo cada vez más datos para demostrar que la psicoterapia es una poderosa intervención que incide sobre el cerebro. Los cambios que la psicoterapia puede introducir a nivel cerebral se comprenden mejor si se los examina a la luz de la neurobiología del desarrollo. Actualmente existe un amplio consenso acerca de que el cerebro del niño es moldeado por el ambiente y las relaciones de apego. Esta necesidad de los estímulos ambientales apropiados para que el sistema nervioso pueda desarrollarse y madurar adecuadamente hace que, desde hace un tiempo, el cerebro sea considerado un órgano bio-ambiental o biosocial (15). 


\section{ENFOQUES PSICOTERAPÉUTICOS}

La psicoterapia es una práctica diseñada para proveer alivio sintomático y cambios en la personalidad, prevenir recaídas y futuros episodios sintomáticos, mejorar la calidad de vida, promover el funcionamiento adaptativo en lo laboral y/o académico y en las relaciones interpersonales, aumentar las probabilidades de tomar decisiones saludables para la vida y ofrecer beneficios establecidos por la colaboración entre el paciente y el terapeuta $(16,17)$.

Los distintos enfoques psicoterapéuticos desarrollados, hasta la actualidad, brindan resultados y muestran evidencias, y fortalecen los fundamentos técnicos que lo sustentan. En cada modelo existen elementos comunes (descritos de manera explícita o de manera implícita en sus postulados iniciales), y que pueden resumirse de la siguiente manera: a. Una cierta conceptualización del comportamiento humano "normal" o "sano"; b. Otra relativa al comportamiento humano "no normal"; y c. Una metodología específica a seguir para generar los cambios deseados.

Además del desarrollo de distintas concepciones teóricas, las investigaciones y la utilización de numerosas técnicas han contribuido a delinear las diversas modalidades terapéuticas, según se han ido privilegiando los factores intrapsíquicos, interpersonales o socio-culturales, tanto en la explicación de la génesis como en cuanto a los objetivos propuestos en la psicoterapia.

Desde el punto de vista de los enfoques desarrollados, basados en distintos paradigmas, es decir el tipo de proceso considerado dominante en la generación de patologías, se destacan los siguientes (18):

- Enfoque psicoanalítico;

- Enfoque cognitivo-conductual;

- Enfoque sistémico; $y$,

- Enfoques de tercera generación.

En los siguientes apartados se describirán las características principales de estos modelos.

\section{Enfoque psicoanalítico}

El término "psicoanálisis" es un neologismo creado por Sigmund Freud para definir un proceso de investigación de los procesos psíquicos inconscientes y un método terapéutico basado en esta investigación.

Existe una gran diversidad de corrientes teóricas dentro del psicoanálisis; no obstante, se podría afirmar que todas coinciden en el concepto de inconsciente, de conflicto psíquico y en la importancia de las experiencias infantiles y del complejo de Edipo como determinantes de las patologías que afectan a los pacientes (18).

La corriente freudiana original plantea, a su vez, más de una concepción del psiquismo, como son la primera y segunda tópica (Tablas 1 y 2$)(18,19)$ : 
TABLA 1. PRIMERA TÓPICA DE FREUD

\begin{tabular}{|c|l|}
\hline Consciente & $\begin{array}{l}\text { Los contenidos conscientes responden a las leyes de la lógica y están gober- } \\
\text { nados por el principio de realidad, por lo que buscan la adaptación al mundo } \\
\text { exterior. }\end{array}$ \\
\hline Inconsciente & $\begin{array}{l}\text { Los contenidos inconscientes sólo pueden ser inferidos, ya que el sujeto no } \\
\text { es consciente de ellos. No les afectan las leyes de la lógica ni categorías como } \\
\text { el tiempo y el espacio. El inconsciente freudiano está constituido por conte- } \\
\text { nidos reprimidos. Estos últimos son representaciones de impulsos o deseos } \\
\text { que serían amenazantes o inaceptables para el sujeto. }\end{array}$ \\
\hline Preconsciente & $\begin{array}{l}\text { Los preconscientes son contenidos inconscientes pero que en un momento } \\
\text { dado pueden transformarse en conscientes porque no son reprimidos por el } \\
\text { sujeto. }\end{array}$ \\
\hline
\end{tabular}

El propósito del psicoanálisis consistiría, entonces, en la ampliación del psiquismo consciente a costa del inconsciente y preconsciente (2).

Freud publica, en una segunda etapa comprendida entre los años 1914 y 1939, "El Yo y el Ello" en el que describe su segunda tópica, a través de la cual expone la estructura del aparato psíquico.

\section{TABLA 2. SEGUNDA TÓPICA DE FREUD}

\begin{tabular}{|c|l|}
\hline Ello & $\begin{array}{l}\text { Parte primitiva, desorganizada e innata de la personalidad. Representa los } \\
\text { impulsos, necesidades y deseos más elementales. Opera de acuerdo con el } \\
\text { principio del placer y desconoce las demandas de la realidad (comida, agresi- } \\
\text { vidad, placer sexual, entre otros). }\end{array}$ \\
\hline Súper Yo & $\begin{array}{l}\text { Contrarresta al Ello. Representa los pensamientos morales y éticos recibidos } \\
\text { de la cultura. Capacidad para la autoevaluación, la crítica y el reproche. }\end{array}$ \\
\hline Yo & $\begin{array}{l}\text { Tiene como fin cumplir de manera realista los deseos y demandas del Ello con } \\
\text { el mundo exterior, a la vez conciliándose con las exigencias del Súper Yo. El } \\
\text { Yo sigue al principio de realidad, satisfaciendo los impulsos del Ello de una } \\
\text { manera apropiada. }\end{array}$ \\
\hline
\end{tabular}

Asimismo, la teorización freudiana reformuló la teoría de las pulsiones: distinguiendo las pulsiones sexuales de las de autoconservación y, posteriormente, las pulsiones de vida a las de muerte. Alrededor de la mitad del siglo XX, las teorías presentadas por Melanie Klein (19) fueron ampliamente asimiladas en el cono sur americano. Estas teorías proponían un modelo de desarrollo temprano centrado en fenómenos iniciales de escisión y proyección, seguidos por movimientos de integración tanto del sujeto como del objeto, y que dieron paso posteriormente a formas más maduras del complejo de Edipo y del funcionamiento psíquico. En esta concepción, la pulsión de muerte y los fenómenos de agresión cumplen un papel importante. Las ideas "kleinianas" se aplicaron en el tratamiento de niños, grupos, pacientes psicóticos y muchos otros (2). 
La teoría psicoanalítica ha sufrido importantes desarrollos a lo largo del siglo XX, con la aparición de nuevos modelos que abogan por distintas dinámicas psíquicas para explicar los síntomas; se podría afirmar que existe una suerte de pluralismo dentro de las principales corrientes psicoanalíticas, sin desmedro de la existencia de una base común (tanto teórica como técnica).

En resumen, además de un conjunto de postulados teóricos sobre el psiquismo humano, el psicoanálisis es un método psicoterapéutico que busca investigar la conflictiva infantil inconsciente del paciente como forma de enriquecimiento en la comprensión de sí mismo y de su historia y concomitantemente como un camino de resolución de su patología.

Este método propone una serie de reglas técnicas que permiten la toma de conciencia de estas influencias primitivas, su transformación y un manejo más libre de las mismas por parte del paciente. Esto se basa en (18-20):

- La asociación libre del paciente, que facilita la regresión y permite que el mismo pueda dar la expresión más amplia a sus emociones y sus pensamientos; $y$,

- La jerarquización de los aspectos no verbales de la comunicación, así como los distintos comportamientos del paciente.

Como contrapartida, el terapeuta debe lograr una actitud de atención libremente flotante, que le permita descubrir configuraciones latentes que pueden no coincidir con el contenido manifiesto del discurso del paciente; asimismo, debe prestar atención a los síntomas, sueños, lapsus, recuerdos y otros. Además, el terapeuta debe mantenerse neutral y no tomar partido por los distintos deseos o fuerzas en conflicto dentro del paciente (19).

La interpretación y la construcción son las herramientas que permiten procesar de una manera mejor los conflictos perturbadores. El terapeuta también debe observar la transferencia (lo que el terapeuta genera en el paciente), que pone de manifiesto los modos de relación primarios reeditados en el vínculo con él y su propia contratransferencia (esto es, sus reacciones involuntarias ante el paciente y lo que este último le genera). Finalmente, el encuadre está dado por el conjunto de constantes de lugar, tiempo, frecuencia, etcétera, que tienden a facilitar que se desarrolle un proceso de cambio en el paciente (2), a través de la comprensión de aspectos inconscientes de sí mismo (insight).

Si bien su autor las concibió para el abordaje de trastornos neuróticos, y no de trastornos psicóticos, muy variadas situaciones clínicas pueden ser objeto de las psicoterapias dinámicas. En general, no hay exclusión por el tipo de patología que padece el paciente, sino que la indicación de dependerá de factores relativos a la capacidad de la persona para beneficiarse de un enfoque psicoanalítico, de su motivación, de su insight y otros.

\section{Enfoque cognitivo-conductual}

Epícteto, filósofo griego que vivió entre los siglos I y II después de Cristo, afirmó "Lo que perturba no son los hechos, sino lo que pensemos sobre ellos". Esta podría ser una precoz referencia que, muchos siglos más tarde, se incluirá dentro del enfoque cognitivo-conductual.

La terapia cognitivo-conductual se desarrolló a partir de la terapia conductual tradicional. Fueron autores importantes del conductismo tradicional, Joseph Wolpe, de Sudáfrica, y Hans Jürgen Eysenck, del Reino Unido. Albert Bandura, de Canadá, también ha influido de modo 
significativo. Además, varios otros autores de orientación cognitiva han proporcionado una base empírica y teórica firme al modelo cognitivo-conductual. Entre los autores de mayor influencia en el desarrollo de este enfoque se encuentran Aaron Beck (terapia cognitiva de las emociones) y Albert Ellis (terapia racional emotiva).

La terapia cognitivo-conductual se refiere a una clase de intervenciones que comparten la misma básica proposición de que los trastornos mentales y el distrés psicológico son mantenidos por factores cognitivos.

La premisa nuclear de esta aproximación terapéutica sostiene que las cogniciones maladaptativas contribuyen a mantener el distrés emocional y los problemas conductuales (21-23).

La terapia racional emotiva de Albert Ellis propone descubrir las ideas irracionales que tiene el paciente y con ello sanar las emociones dolorosas, dramatizadas y exageradas, que son consecuencia de los esquemas mentales distorsionados (23). La tabla 3 expone las tres principales creencias irracionales formuladas por Ellis (24).

\section{TABLA 3. LAS TRES CREENCIAS IRRACIONALES BÁSICAS}

\begin{tabular}{|c|l|}
\hline Con respecto a uno mismo & $\begin{array}{l}\text { Idea irracional: "Debo hacer las cosas bien y merecer la aprobación de los de- } \\
\text { más por mis actuaciones". }\end{array}$ \\
\hline $\begin{array}{c}\text { Con respecto a los demás } \\
\text { Con respecto a la vida } \\
\text { (o al mundo) }\end{array}$ & $\begin{array}{l}\text { Idea irracional: "Los demás deben actuar de manera considerada, agradable } \\
\text { justacional: "La vida debe ofrecerme unas condiciones buenas y fáciles } \\
\text { para que pue conseguir lo que quiero sin mucho esfuerzo ycon comodidad". }\end{array}$ \\
\hline
\end{tabular}

Las investigaciones de Ellis sostienen que no son los acontecimientos (A) los que generan estados emocionales (C), sino la manera de interpretarlos (B), a través de creencias irracionales.

Por tanto, si el paciente es capaz de modificar sus esquemas mentales, será -a su vez- capaz de generar nuevos estados emocionales menos dolorosos, más racionales y realistas.

Una visión general de la terapia racional emotiva de Ellis puede encontrarse en la figura 1 (23, 24): 
Figura 1. Visión general de la terapia racional emotiva.

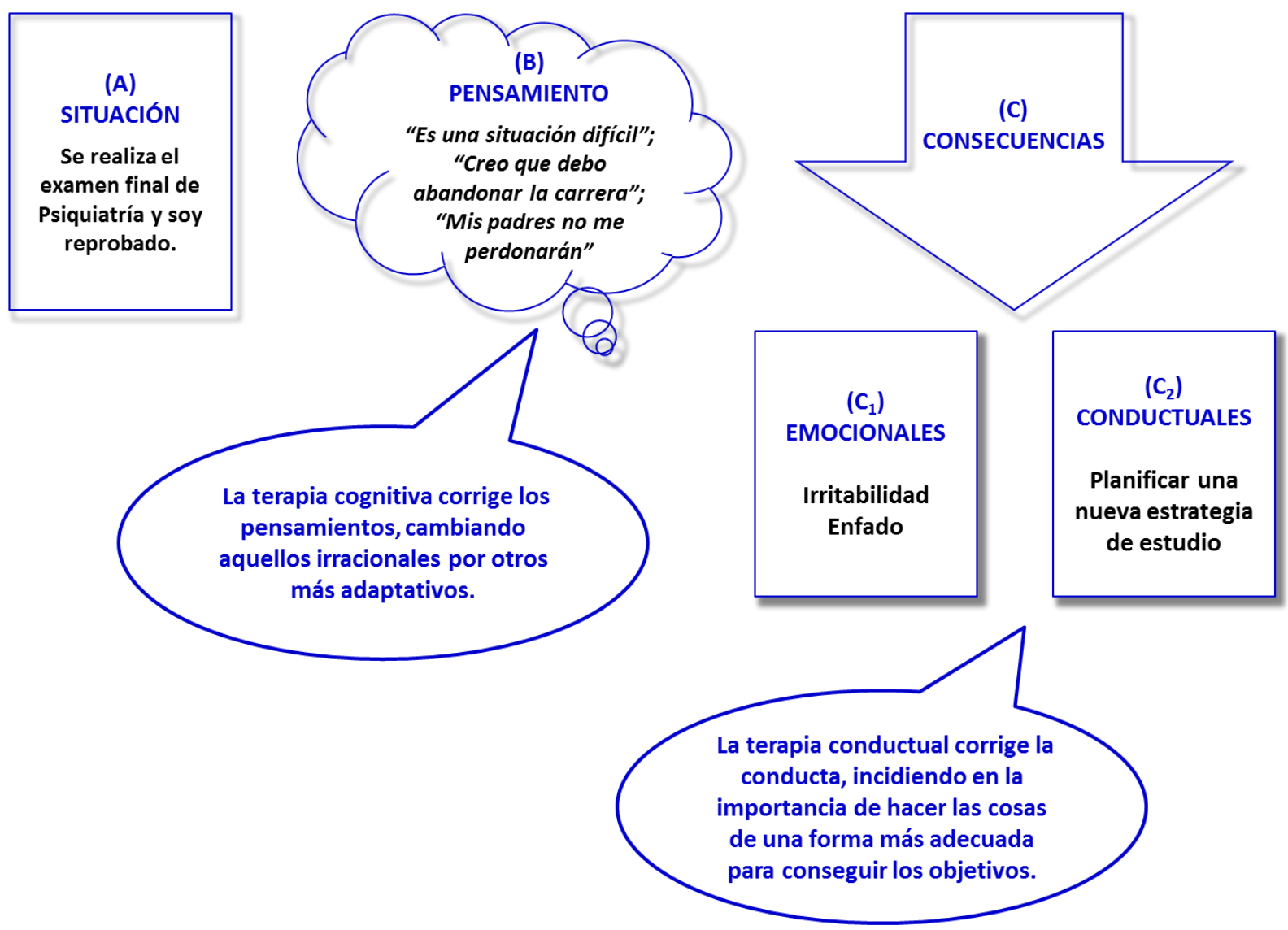

Por su parte, según el modelo de Aaron Beck, las cogniciones maladaptativas incluyen creencias generales, o esquemas, sobre el mundo, el yo, y el futuro, dando lugar a la aparición de pensamientos específicos y automáticos en situaciones particulares. El modelo básico centra su estrategia terapéutica en modificar estas cogniciones maladaptativas, logrando cambios en el distrés emocional y los problemas conductuales (22).

El procesamiento de la información es una característica que define al ser humano, y que permite a los individuos realizar representaciones de sí mismos y de su mundo. El ser humano está en un continuo procesamiento de información, tanto del interior como del exterior, recibiendo, codificando, interpretando, almacenando y recuperando dicha información, con un papel fundamental en la adaptación y la supervivencia (18).

Por tanto, se podría afirmar que la respuesta ante un determinado acontecimiento vital está condicionada por los esquemas cognitivos que tiene la persona (Beck) o por sus creencias irracionales (Ellis). Estas asunciones o supuestos son adquiridos en etapas tempranas de la vida, y permanecen a nivel no consciente, activándose posteriormente por diversos eventos y generando determinadas interpretaciones que desfiguran dichos acontecimientos (distorsiones cognitivas) y originan problemas emocionales, conductuales y relacionales. 
Componentes de la terapia cognitivo-conductual

Beck afirma que el cambio en el modo de procesar e interpretar la información, así como de los esquemas cognitivos que resultan maladaptativos, mejora el malestar psicológico. En resumen, la terapia cognitivo-conductual enseña al paciente a identificar, evaluar y responder a los pensamientos y creencias disfuncionales, empleando diversas técnicas para modificar esos pensamientos, el estado de ánimo y los comportamientos (22).

\section{Pensamientos automáticos}

Ocurren espontáneamente, por reflejo, y no como resultado de una reflexión o razonamiento sobre la situación. Aunque parecen plausibles y razonables, no siguen una lógica de pasos como ocurre en la resolución de problemas. Son reflejo de suposiciones y creencias esenciales de las personas, pueden ocurrir a pesar de evidencias objetivas de lo contrario y aumentan la intensidad de la emoción.

El paciente puede realizar un informe de autorregistro de pensamientos automáticos, que acompañan a las reacciones emocionales, a fin de mostrarle las distorsiones cognitivas que utiliza, facilitando de esa manera su cambio. Los pensamientos automáticos son el resultado de distorsiones cognitivas, algunas de las cuales son (18):

1. Inferencia arbitraria: Extracción de conclusiones en ausencia de evidencia.

2. Sobregeneralización: Tendencia a extraer conclusiones en base a uno o más incidentes aislados.

3. Abstracción selectiva: Focalización en un detalle fuera de contexto, irrelevante, ignorando hechos más importantes.

4. Pensamiento dicotómico: Tendencia a clasificar las ideas en categorías (o polaridades) extremas.

5. Personalización: Tendencia a relacionar algo del ambiente consigo mismo.

\section{Supuestos/creencias intermedias}

Creencias o ideas profundas que tiene el paciente sobre sí mismo, sobre los demás y el mundo. Las creencias intermedias dan lugar a los pensamientos automáticos.

\section{Creencias centrales}

Son ideas globales, rígidas y absolutas sobre uno mismo y sobre los demás. Se desarrollan a lo largo de la infancia y son las ideas más nucleares que tiene una persona sobre sí misma.

El modelo cognitivo-conductual puede resumirse en la figura $2(21,22)$. 
Figura 2. El modelo cognitivo-conductual.

\section{EL MODELO COGNITIVO}

Creencias centrales

Soy incompetente

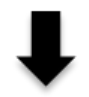

Creencias intermedias

Si no comprendo algo de manera perfecta, soy un tonto

Situación

Leyendo el libro de Psiquiatría

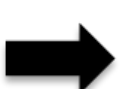

"Esto es muy difícil"; "Jamás lo entenderé"

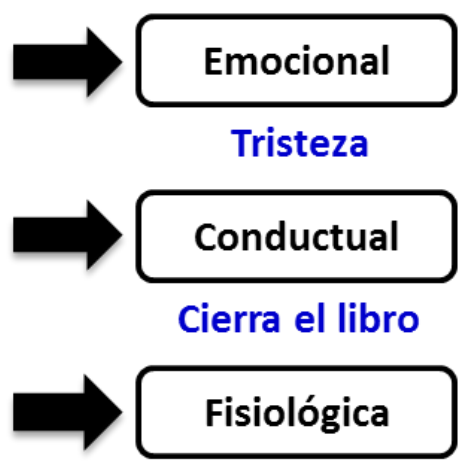

Pesadez en el abdomen

En el enfoque cognitivo-conductual se emplean muchas técnicas, tanto conductuales como cognitivas. Merece especial destaque la técnica de la reestructuración cognitiva. Sucintamente, esta técnica se basa en la identificación del pensamiento automático, en el reconocimiento de la emoción y la conducta asociada a ese pensamiento, el posterior cuestionamiento del pensamiento por parte del paciente y, finalmente, la sustitución de lo maladaptativo por un pensamiento alternativo.

Las psicoterapia cognitivo-conductual busca establecer estrategias diferenciadas para los distintos trastornos psicológicos. Actualmente, es posible tratar con este modelo toda la patología mental, tanto en forma individual como grupal (2).

Por último, se destaca que la terapia cognitivo-conductual es el modelo más contrastado empíricamente (16) y uno de los más ampliamente aceptados por la comunidad científica (véase más adelante en este mismo artículo). 


\section{Enfoque sistémico}

La principal característica del enfoque sistémico es que centra sus objetivos terapéuticos en la modificación de patrones de interacción entre las personas (25). Históricamente el desarrollo de los modelos clínicos sistémicos ha estado unido a la evolución de la terapia familiar. Sin embargo, actualmente el campo de aplicación es mucho más amplio y abarca también tratamientos individuales, grupales, de pareja y, obviamente, también de familias.

En la década de los 60 del siglo pasado, los autores sistémicos se alejaron de los planteamientos psicoanalíticos, centrándose en la teoría de la comunicación. En Estados Unidos de América, Bateson publicó su teoría del doble vínculo que explica la esquizofrenia como un intento límite para adaptarse a un sistema familiar con estilos de comunicación incongruentes o paradójicos, mientras que Minuchin postuló que los sistemas familiares se organizan como alianzas (mayor cercanía afectiva entre dos miembros de la familia) o coaliciones (alianzas de dos en contra de un tercero) $(18,26)$.

En el modelo sistémico, en general, los síntomas son entendidos como expresiones disfuncionales del sistema familiar, de modo que el foco de análisis no recae solamente en el paciente que tiene el síntoma o problema, sino que abarca a todo el sistema.

Al paciente que tiene las manifestaciones sintomáticas o el problema concreto se lo denomina paciente identificado. Todas las escuelas ponen especial interés en las conductas y en los patrones de intercambio entre los miembros que componen el sistema, es decir, los integrantes de la familia. Se focaliza en general en las interacciones y pautas repetidas que marcan un estilo de vinculación entre los miembros. La solución de los problemas o síntomas, se encuentran en estrecha vinculación con las relaciones y pautas que establecen los miembros del sistema entre sí, por eso se trabaja fundamentalmente con los patrones disfuncionales de interacción familiar (25).

La terapia sistémica posee un variado repertorio de métodos altamente eficaces, destinados a satisfacer las necesidades del paciente y su familia (Tabla 4) (27).

\section{TABLA 4. MÉTODOS DE LA TERAPIA SISTÉMICA}

1. Un conocimiento de cómo operan los procesos familiares y la habilidad de que estos procesos sean evidentes para la familia;

2. Una capacidad de trabajar con los niños en la relación con sus padres y viceversa;

3. Trabajo con las familias para comprender y utilizar productivamente la influencia de sus antecedentes y tradiciones;

4. Tanto a través de la conversación como de la acción, ayudar a los miembros de la familia a reconocer opciones no tenidas en cuenta previamente; $y$,

5. Explorar de manera colaborativa las fortalezas y recursos que tienen los miembros de la familia, y que pueden ser utilizados para apoyarse mutuamente.

El enfoque sistémico, como se expresó más arriba, puede emplearse en el contexto individual, de pareja, familiar, sistemas profesionales y otros más amplios. Generalmente es ofrecido a parejas o familias, pero siempre teniendo en mente los sistemas, pequeños y grandes, en los que están insertos, a fin de tomar conciencia de que cualquier cambio a cualquier nivel del sistema seguramente impactará globalmente. Se debe hacer notar que el enfoque sistémico, 
actualmente, ha incorporado el entendimiento de los procesos internos del individuo, tal como lo hacen los enfoques psicoanalítico y cognitivo-conductual (27). Sus indicaciones abarcan las dificultades de la familia por resolver demandas de crecimiento y diferenciación de los hijos, problemas planteados a partir del divorcio y o nuevas uniones, abuso de sustancias y trastornos mentales (2).

\section{Enfoques de tercera generación}

Hace poco más de 10 años, Hayes diferenció tres generaciones de terapias (28). La primera generación se refiere a la terapia de conducta clásica apoyada en el cambio directo del comportamiento mediante el manejo de contingencias. A pesar del avance trascendental que supuso el elenco de procedimientos y éxitos conseguidos (actualmente vigentes), no fue eficaz para el tratamiento de ciertas problemáticas. Se alentó entonces en la necesidad de centrarse sobre la dimensión cognitiva y se formalizó el enfoque que hoy se conoce como terapia cognitivo-conductual. Éstas conforman la segunda generación de terapias, que asumieron las técnicas centradas en el cambio por contingencias pero otorgando un papel primordial a los eventos cognitivos como eje causal y mecánico del comportamiento. Postulan su tratamiento directo para poder modificar el comportamiento del paciente. Estas terapias han resultado exitosas pero presentan una serie de limitaciones (29).

El problema principal es que la explicación y los modos de intervención sobre los problemas que estas técnicas ofrecen son funcionalmente equivalentes a los establecidos culturalmente, aunque se presenten con ropajes especiales. Sin embargo, no han proporcionado, completamente, una base experimental sobre la formación, derivación y alteración de los eventos privados, ni de las condiciones en las que se establecen y cambian las relaciones entre los eventos cognitivos y las acciones, ni las bases experimentales sobre las que se fundamentan la mayoría de los métodos clínicos.

No obstante a lo anterior, lo cierto es que la terapia cognitivo-conductual goza de buena salud siendo la terapia que más réditos ha cosechado en el ámbito de los tratamientos psicoterapéuticos con adultos $(21,28-30)$. Este entendimiento estándar ampliamente diseminado sobre el funcionamiento del ser humano por las terapias de segunda generación (y compartido por las terapias farmacológicas) implica que las acciones de la persona están reguladas por sus pensamientos y emociones, de modo que para cambiar el funcionamiento ineficaz se ha de controlar de algún modo aquello que genere malestar, y el malestar mismo. Por ello, las terapias de segunda generación van dirigidas al cambio de los eventos cognitivos como un medio para alterar las acciones de la persona que presenta trastornos mentales.

El apogeo de las terapias de tercera generación se dio, entre otros motivos, por un desconocimiento sobre por qué funciona o fracasa la terapia cognitiva-conductual y por la existencia de concepciones principalmente funcionales del comportamiento humano (28-31).

La tercera generación de terapias representa, para muchos autores, un salto cualitativo porque las técnicas que engloba están orientadas, no a la evitación/reducción de síntomas, sino a que la persona actúe con la responsabilidad de la elección personal y la aceptación de los eventos privados que conlleve ese proceder. Entre estas terapias figuran, entre otras, la terapia conductual dialéctica de Linehan (1993), la terapia de aceptación y compromiso de Hayes, Stroshal y Wilson (1999), y la terapia cognitiva basada en mindfulness de Seagal, Williams y 
Teasdale (2002) (32-34). Todas estas terapias han sido utilizadas por psiquiatras y psicólogos para el manejo de diversas patologías, entre las que destacan los trastornos de la personalidad (35).

Es importante destacar que a los enfoques de tercera generación los caracteriza una modalidad diferente de relacionarse con los contenidos; no se busca cuestionar las creencias disfuncionales, mediante el análisis lógico, sino que se busca modificar los procesos cognitivos, particularmente la atención.

\section{Terapia conductual dialéctica}

La terapia conductual dialéctica fue propuesta por la psicóloga estadounidense Marsha Linehan para el tratamiento de mujeres con historial de intentos de suicidio, ideación suicida y automutilaciones. Linehan utilizaba la terapia cognitivo conductual para enfrentarse a dichos casos antes de elaborar su propuesta $(33,36)$; no obstante, continuamente se encontraba con dificultades relacionadas con la imposibilidad del manejo de las manifestaciones clínicas en conjunto con el desarrollo de sesiones terapéuticas, dirigidas a la implementación de estrategias adaptativas de afrontamiento (37).

La terapia conductual dialéctica parte de las conceptualizaciones en torno al trastorno límite de la personalidad, el cual se caracteriza por un patrón generalizado de inestabilidad en las relaciones interpersonales de la persona y su autoimagen, así como, una marcada impulsividad manifestada en diversos contextos, instaurándose como la primera propuesta de intervención en demostrar su eficacia en el tratamiento de este tipo de problemáticas (37). Dentro de esta perspectiva, el trastorno límite de la personalidad es concebido como una disfunción del sistema de regulación emocional del individuo por encontrarse en interacción con un ambiente caracterizado por la invalidación y que provocará como consecuencia, una desregulación emocional expresada en inhabilidad para modular las emociones, implicando una alta vulnerabilidad emotiva que hace que la persona sea mucho más sensible a los estímulos, por lo que vive los sentimientos de manera mucho más intensa (38).

Las personas con conductas propias del trastorno límite de la personalidad tienen como consecuencia una respuesta emocional intensificada que puede ser el resultado de vulnerabilidades biológicas, consecuencia de experiencias traumáticas y/o negligencia vivida durante la infancia temprana, provocando cambios en las estructuras neuronales encargadas de la regulación emocional. Bajo estas circunstancias, la respuesta del individuo a estímulos ambientales se caracteriza por una mayor velocidad e intensidad que en otros (36). En este complejo contexto clínico, la terapia conductual dialéctica surge como una alternativa para el tratamiento de personas con dicha sintomatología y diagnóstico, empleando técnicas orientadas hacia la búsqueda del cambio conductual, en conjunto con intervenciones que giran alrededor de la aceptación y validación del comportamiento (39).

La terapia conductual dialéctica asume que estos individuos carecen de habilidades interpersonales y regulatorias básicas, y que factores personales y ambientales frecuentemente bloquean y/o inhiben la utilización de habilidades conductuales, reforzando entonces conductas desadaptativas en la persona (40).

La terapia conductual dialéctica, como se mencionó, emplea algunas de las estrategias de la terapia cognitivo-conductual para el tratamiento de las problemáticas relacionadas con las 
personas con diagnóstico de trastorno límite de la personalidad, centrándose principalmente en el entrenamiento de habilidades, el manejo de contingencias, la reestructuración cognitiva y las técnicas de exposición. Estos procedimientos a su vez, son enriquecidos por medio de la incorporación de estrategias para aumentar el compromiso con la terapia, estrategias de aceptación y estrategias dialécticas (41).

\section{Terapia de aceptación y compromiso}

La terapia de aceptación y compromiso combina estrategias de aceptación y toma de consciencia del comportamiento propio por parte de los individuos como una forma de consecución del cambio conductual, por lo que la puesta en práctica de dichas destrezas va a influir directamente en la mejora de la "flexibilidad psicológica" de los consultantes. La flexibilidad psicológica constituye la habilidad de la persona para entrar en contacto con el presente de una forma más completa como ser humano consciente y a partir de dicho contacto, se decide cambiar el comportamiento problemático o persistir en el comportamiento que se considera necesario para alcanzar metas (42-44).

El proceso terapéutico en la terapia de aceptación y compromiso tiene como premisa fundamental que la experiencia de dolor psicológico es inevitable y que la utilización de la evitación de las experiencias y demás mecanismos de afrontamiento similares para lidiar con el malestar, usualmente incrementan el sufrimiento en las personas aunque sea comprensible su empleo (45). La persona cuando inicia el proceso de intervención psicoterapéutica se encuentra atrapada en el patrón de evitación, en una suerte de lucha inquebrantable por dejar de estar en dicho patrón. Este esquema se muestra como un callejón sin salida, debido a que la lucha por eliminar o reducir el malestar hace que las acciones llevadas a cabo dirijan a la persona a otro estado de malestar continuo, por lo que la persona siempre se encuentra experimentando sufrimiento psicológico (42).

La tabla 5 resume los objetivos generales de la terapia de aceptación y compromiso $(42,43)$.

TABLA 5. OBJETIVOS GENERALES DE LA TERAPIA DE ACEPTACIÓN Y COMPROMISO

1. La clarificación de los valores de la persona consultante;

2. La aceptación de los eventos privados de la persona consultante, principalmente relacionados con aquellos acontecimientos que no pueden modificarse y que consecuentemente va a implicar el abandono de conductas de afrontamientos no efectivos y el saber escoger flexiblemente el comportamiento funcional y efectivo; $y$,

3. El fortalecimiento del "yo como contexto" para que la persona consultante sea capaz de contemplar sus eventos privados y a partir de la reflexión, elegir el camino más adecuado para sus propios intereses.

Como se puede observar, los objetivos de la intervención se encuentran focalizados en la propiciación del cambio a través de la aceptación de las particularidades propias del individuo, tomando una actitud crítica ante aquellos elementos de la experiencia subjetiva que no pueden ser modificados y haciendo esfuerzos por adoptar estrategias de afrontamiento más constructivas para aquellos factores que tienen amplias probabilidades de cambio. 
Terapia cognitiva basada en "mindfulness"

La terapia cognitiva basada en mindfulness (también llamada terapia cognitiva basada en la atención plena) es una integración de la terapia cognitiva con el programa de reducción del estrés de Jon Kabat-Zinn $(46,47)$. Fue desarrollada inicialmente para afrontar el estrés, el dolor y la enfermedad.

El mindfulness (la atención plena) se define como una habilidad que consiste en centrar la atención de un modo intencional en un objeto, en el momento presente, y sin juzgar (46). Los humanos son, normalmente, poco conscientes de su momento a momento, puesto que en general actúan en "piloto automático"; aun así el estado de mindfulness es un estado común que todas las personas han sentido alguna vez y que son capaces de desarrollar.

El desarrollo del mindfulness es gradual, progresivo y requiere práctica regular. Lo más frecuente es realizar esta práctica a través de la meditación. La capacidad de darse cuenta de las experiencias momento a momento genera un sentido de la vida más rico y completo. Así, al observar persistentemente, sin juzgar, el contenido mental, gradualmente aumenta la capacidad las personas de percibir sus propias respuestas mentales a estímulos (externos e internos), lo que se decanta en un aumento de las acciones afectivas en el mundo y por tanto un aumento de la percepción de control (48).

Antes de explicar brevemente este modelo psicoterapéutico, se hace necesario recordar algunos aspectos básicos de la terapia cognitiva de Aaron Beck. Ésta plantea que cualquier influencia sobre la cognición debería seguirse de un cambio en el estado del ánimo y, en consecuencia, de modificaciones a nivel de la conducta. La teoría sugiere que el cambio puede producirse indistintamente en cualquiera de las áreas que se incida: sobre la emoción, el pensamiento o la conducta. Sin pretender dar una explicación etiológica completa de los trastornos del ánimo, el profesor Beck consideró la cognición disfuncional como una cuestión central de la patología (49).

En la terapia cognitiva basada en mindfulness se entrena la capacidad de la persona de redirigir la atención hacia cogniciones que rompan con engranajes desadaptativos, que pueden, por ejemplo, activar una recaída depresiva. El objetivo de esta terapia es crear un tratamiento grupal que entrene la atención y que ofrezca nuevos esquemas mentales y formas de afrontar a los propios pensamientos, impidiendo así el acceso a esquemas disfuncionales. Se ayuda así a los pacientes a liberarse de procesos rumiativos y autoperpetuados cuando se sienten tristes, ya que detrás de estos procesos existe siempre una discrepancia entre el estado actual ("cómo se está") y el estado deseado ("cómo se debería estar") y la distancia que los separa (46).

La esencia del mindfulness es la utilización intencionada de la atención para establecer una configuración del engranaje desadaptativo. El entrenamiento en atención plena aumenta la capacidad metacognitiva de descentramiento, es decir, la capacidad de tomar perspectiva, y permite a los pacientes usarla cuando sus pensamientos y sentimientos están fuera de control o cuando se activan estilos cognitivos disfuncionales como la rumiación o la preocupación. El tratamiento tiene una duración, por lo general, de 8 sesiones, cada una de dos horas de dura- 
ción. En las mismas se enseña a meditar en grupo, y se trabajan las tareas para el hogar (autorregistros de pensamientos, sentimientos y emociones; práctica de meditación, entre otros) $(46,47)$.

\section{EFICACIA DE LA PSICOTERAPIA}

La eficacia de la psicoterapia es aceptada ampliamente como significativa (6). Esta eficacia está en consonancia a través de la mayoría de los diagnósticos, con variaciones más influenciadas por la severidad del cuadro que por el diagnóstico en particular. Esto es, las variaciones observadas en los resultados están más afectadas por las características particulares del paciente (cronicidad del cuadro, complejidad de la patología, severidad, nivel de apoyo social, del terapeuta y del contexto antes que por el diagnóstico particular o modalidad psicoterapéutica utilizada $(50,51)$.

Más aún, muchas investigaciones afirman que los resultados a largo plazo de la psicoterapia tienden a durar más y a necesitar menos refuerzos terapéuticos adicionales que, inclusive, los tratamientos psicofarmacológicos. Por ejemplo, en el tratamiento de la depresión y de los trastornos del espectro ansioso, los pacientes adquieren una variedad de habilidades que pueden ser utilizadas luego de la finalización del tratamiento psicoterapéutico y, generalmente, pueden continuar mejorando luego de finalizar el mismo $(52,53)$.

Para la mayoría de los trastornos mentales, la evidencia de estudios clínicos rigurosos demuestra que una variedad de enfoques psicoterapéuticos es efectiva con niños y adolescentes, adultos y adultos mayores. Estos estudios, por lo general, muestran significativos efectos beneficiosos de la psicoterapia en comparación a no recibir tratamiento alguno (6). En el caso del tipo de psicoterapia específica, no existen mayores diferencias, observándose que los diferentes enfoques producen, típicamente, resultados similares $(54,55)$.

En el caso específico de la terapia cognitivo-conductual, se ha comparado la eficacia de la misma con otras terapias (tratamiento farmacológico, tratamiento combinado de terapia cognitivo-conductual y psicofármacos, placebo farmacológico, placebo psicológico, otro tratamiento psicoterapéutico), en algunos de los trastornos mentales más frecuentes, mediante una revisión sistemática Cochrane (2003-2010) y resultados de metaanálisis y estudios controlados aleatorizados. Las principales conclusiones del estudio reflejaron (56):

1. La terapia cognitivo-conductual es de primera elección para muchos trastornos mentales no psicóticos (adultos, niños y adolescentes);

2. En todos los trastornos, la terapia cognitivo conductual fue superior al placebo farmacológico y al placebo psicológico; $y$,

3. En comparación con otro tipo de tratamiento psicoterapéutico, la terapia cognitivo conductual fue superior, salvo resultados no concluyentes en el trastorno depresivo mayor.

Asimismo, Hofmann y colaboradores (21) analizaron 106 metaanálisis, a fin de examinar la eficacia de la terapia cognitivo conductual en los siguientes trastornos/problemas: trastorno por uso de sustancias, esquizofrenia y otros trastornos psicóticos, depresión y distimia, trastorno bipolar, trastornos de ansiedad, trastornos somatomorfos, trastornos de la conducta alimentaria, insomnio, trastornos de la personalidad, ira y agresividad, conductas criminales, estrés, distrés secundario a enfermedades médicas, dolor crónico y fatiga, distrés secundario 
a complicaciones del embarazo y trastornos hormonales en mujeres. Algunas de las conclusiones más importantes pueden leerse en la tabla 6.

\section{TABLA 6. EFICACIA DE LA PSICOTERAPIA}

\begin{tabular}{|c|l|}
\hline $\begin{array}{c}\text { Adicciones y trastornos } \\
\text { por uso de sustancias }\end{array}$ & $\begin{array}{l}\text { La eficacia de la terapia cognitivo conductual es de pequeña a mediana, de- } \\
\text { pendiendo del tipo de sustancia. No obstante, es altamente efectiva para el } \\
\text { tratamiento de la dependencia a cannabis y a nicotina }(21,57) .\end{array}$ \\
\hline Esquizofrenia & $\begin{array}{l}\text { Los estudios reflejan la eficacia de la terapia cognitivo conductual en la remi- } \\
\text { sión de la sintomatología positiva (ideas delirantes, alucinaciones) y, además, } \\
\text { es promisoria como terapia adyuvante a la psicofarmacología en pacientes } \\
\text { con un brote agudo. En el caso de pacientes crónicos o para la prevención de } \\
\text { recaídas, la eficacia de la terapia cognitivo-conductual es menor que la psico- } \\
\text { farmacología o la intervención familiar (58). }\end{array}$ \\
\hline
\end{tabular}

\begin{tabular}{|c|c|}
$\begin{array}{c}\text { Trastornos depresivos } \\
\text { y distimia }\end{array}$ & $\begin{array}{l}\text { Los estudios sobre la eficacia de la terapia cognitivo-conductual para la de- } \\
\text { presión y la distimia arrojan resultados contradictorios: algunos sugieren una } \\
\text { alta eficacia y otros, por su parte, insisten en su debilidad (59). }\end{array}$ \\
\hline Trastorno bipolar & $\begin{array}{l}\text { La eficacia de la terapia cognitivo-conductual por sí sola tiene evidencia limi- } \\
\text { tada sobre el enfoque psicofarmacológico. Por lo tanto, se considera que más } \\
\text { estudios deben realizarse acerca de esta modalidad psicoterapéutica y los } \\
\text { trastornos del estado de ánimo (59). }\end{array}$ \\
\hline $\begin{array}{c}\text { Trastornos del } \\
\text { espectro ansioso }\end{array}$ & $\begin{array}{l}\text { La terapia cognitivo-conductual es de primera línea terapéutica en estos tras- } \\
\text { tornos, con efectos positivos significativos no solo en el trastorno primario } \\
\text { sino también en síntomas secundarios de ansiedad, como algunas disfuncio- } \\
\text { nes del sueño (60). }\end{array}$ \\
\hline
\end{tabular}

\begin{tabular}{|c|l|}
\hline $\begin{array}{c}\text { Trastorno obsesivo } \\
\text { compulsivo }\end{array}$ & $\begin{array}{l}\text { Se han demostrado importantes efectos benéficos de la terapia cognitivo- } \\
\text { conductual en el manejo de obsesiones y compulsiones (61). }\end{array}$ \\
$\begin{array}{c}\text { Trastorno de síntomas } \\
\text { somáticos y trastornos rela- } \\
\text { cionados }\end{array}$ & $\begin{array}{l}\text { Eficacia de mediana a grande ha sido evidenciada en el tratamiento de tras- } \\
\text { tornos de síntomas somáticos. La terapia cognitivo conductual es específica- } \\
\text { mente útil para reducir la angustia y el excesivo uso de servicios médicos por } \\
\text { parte de pacientes afectados por este tipo de trastornos (62). }\end{array}$ \\
\hline Bulimia & $\begin{array}{c}\text { La terapia cognitivo conductual es considerablemente más efectiva que otros } \\
\text { enfoques psicoterapéuticos, pero menos estudiada para otros trastornos de } \\
\text { la conducta alimentaria (21). }\end{array}$ \\
\hline Insomnio & $\begin{array}{l}\text { La eficacia de la terapia cognitivo conductual en el tratamiento del insomnio } \\
\text { ha sido bien evidenciada y es una de las mejores formas de tratamiento para } \\
\text { el insomnio a mediano y largo plazo (63, 64), mejorando la calidad del sueño, } \\
\text { tiempo total del mismo y eficiencia del sueño. }\end{array}$ \\
\hline Control de ira y agresividad & $\begin{array}{l}\text { La terapia cognitivo conductual ha demostrado ser mediamente eficaz en el } \\
\text { manejo de problemas derivados de estos signos/síntomas (65). }\end{array}$ \\
\hline
\end{tabular}

Finalizando, existe un creciente cuerpo de evidencia que afirma que los enfoques psicoterapéuticos son costo-efectivos, reducen la discapacidad, morbilidad y mortalidad, mejoran el funcionamiento laboral/académico y disminuyen la necesidad de hospitalizaciones psiquiátri- 
cas (66). Como práctica profesional sanitaria, la psicoterapia es sumamente beneficiosa y excede de sobremanera los beneficios experimentados por pacientes que necesitan de servicios de salud mental, pero que no reciben psicoterapia; por lo tanto, la práctica psicoterapéutica debería ser incluida en los sistemas de salud pública, como una herramienta terapéutica útil, basada en la evidencia.

\section{PSICOTERAPIA EN NIÑOS Y ADOLESCENTES}

Podría decirse que el origen de la psicoterapia infantil se remonta a 1909, año en que Freud publicó el ensayo titulado "La fobia de Juanito".

En esta obra, de forma indirecta a través de su padre, Sigmund Freud analizó los temores de Juanito: un miedo irracional a ser mordido por un caballo. Más tarde, Melanie Klein propuso la utilización del juego como sustitutivo de la verbalización en la terapia infantil. En 1977, Virginia Axline pone de moda la terapia conocida como salón de juegos, cuya variante bautizada como pirámide de Maslow alcanzó mucho prestigio en las siguientes décadas. Asimismo, a partir de ahí, se desarrollaron otros métodos, como el conocido como enfoque Gestalt.

A pesar de que su origen se remonta hace más de 100 años, por mucho tiempo la psicoterapia en la infancia y la adolescencia ha sido concebida como (67):

1. Una intervención de tipo "menor" para abordar los trastornos de la infancia, ya que no eran "graves";

2. Una intervención que excluía otro tipo de abordajes, lo que durante tiempo se vio como un enfrentamiento entre biologicismo y psicologicismo;

3. Una intervención para ser desarrollada por "otros" profesionales que no fueran psiquiatras; y,

4. Una intervención basada en "convencer", "mentalizar", "motivar", "charlar", "caer bien o empatizar" con el niño o adolescente.

No obstante, gracias al denodado trabajo de psicoterapeutas infanto-juveniles (psiquiatras y psicólogos), y en virtud a lo demostrado por la medicina basada en la evidencia, este tipo de concepciones están siendo rebatidas. La psicoterapia en niños y adolescentes sitúa la realidad personal del paciente pediátrico en el centro de su actividad y reivindica una visión humanista de la psiquiatría y la medicina como parte esencial del quehacer terapéutico (68).

\section{Intervenciones psicoterapéuticas en infancia y adolescencia}

En la tabla 7 se resumen los esquemas de intervenciones psicoterapéuticas, propuestas por Pedreira, adaptadas a la infancia y a la adolescencia (67): 
TABLA 7. INTERVENCIONES PSICOTERAPÉUTICAS PARA INFANCIA Y ADOLESCENCIA

\begin{tabular}{|c|c|}
\hline Intervención individual & $\begin{array}{l}\text { Para asumir el sufrimiento del niño o adolescente, la situación del desarrollo } \\
\text { y evolución de los procesos y, finalmente, la maduración emocional y afectiva } \\
\text { de los sujetos. }\end{array}$ \\
\hline Intervención grupal & $\begin{array}{l}\text { Asume la relación con los pares, es decir, con los amigos, compañeros y las } \\
\text { dificultades que aparecen en dicha relación (función del liderazgo, retrai- } \\
\text { miento social, dificultades de comunicación, entre otros). }\end{array}$ \\
\hline Intervención familiar & $\begin{array}{l}\text { Consiste en elaborar la historia familiar a nivel significativo de sus integrantes, } \\
\text { orientar en las dificultades que van apareciendo a lo largo de las distintas cri- } \\
\text { sis familiares. }\end{array}$ \\
\hline Intervención institucional & $\begin{array}{l}\text { Este tipo de intervención tiene su origen en abordar los diferentes lugares en } \\
\text { los que se desarrolla la vida del niño o adolescente. Cuatro son los grupos } \\
\text { sobre los que incidir, con una orientación terapéutica: incrementar la capaci- } \\
\text { dad de discriminación de los problemas que aparecen en los niños; derivación } \\
\text { y su técnica desde una institución a otra, en aquellos casos que precisen aten- } \\
\text { ción; devolución de los contenidos encontrados como compromiso para ase- } \\
\text { gurar una correcta relación interinstitucional y desarrollar un método de con- } \\
\text { tención como fundamento de la intervención conjunta. }\end{array}$ \\
\hline Intervención comunitaria & $\begin{array}{l}\text { Persigue la modificación de actitudes, la movilización y coordinación de los } \\
\text { recursos asistenciales en el territorio. }\end{array}$ \\
\hline
\end{tabular}

\section{Aspectos diferenciales de la psicoterapia infantil}

Si bien en los adolescentes mayores, la psicoterapia toma aristas similares a la empleada en adultos jóvenes, los métodos de psicoterapia en niños más pequeños se encuentran muy influenciados por tres características claves y distintivas en relación a las terapias para adultos (69).

\section{El juego como elemento clave}

Por definición, el lenguaje de los niños es el juego, especialmente en los primeros años de edad. El juego es para los niños el equivalente al lenguaje en los adultos, y a través de éste pueden divertirse, comunicarse, descubrir y explorar el mundo, adquirir conocimientos y también representar sus miedos, deseos, conflictos, preocupaciones y problemas. Los terapeutas especializados tienen la preparación suficiente para interactuar con pacientes de corta edad mediante el juego, detectando sus problemáticas y ayudándoles en su resolución. Desde el juego se pueden trabajar los distintos aspectos del tratamiento y aplicar los aspectos básicos de los principales enfoques psicoterapéuticos.

Implicación de la familia

La psicoterapia infantil se puede realizar junto a los padres, en sesiones mixtas y de forma individual, es decir, a solas entre el terapeuta y el niño. Pero en todos los casos es necesaria la implicación directa de los padres, por lo que se les cita regularmente en sesiones con el doble objetivo de informarles de los avances de su hijo y darles indicaciones de participación 
e intervención: continuación de la terapia en casa, cambios en la relación con sus hijos, necesidad de intensificar la comunicación o los lazos afectivos, entre otros.

\section{Distintos niveles de objetivos}

La influencia de la implicación familiar va más allá de la asistencia o seguimiento de la terapia de sus hijos, puesto que influye también en los objetivos. En la psicoterapia infantil hay que tener en cuenta, por supuesto, las necesidades y problemas de los niños, pero también las esperanzas y expectativas de los propios padres. Es habitual que cada parte, el niño por un lado y sus padres por otro, tengan expectativas distintas en relación al proceso terapéutico y, por lo tanto, una percepción diferente de los logros obtenidos y, en consecuencia, distintos grados de satisfacción sobre el trabajo realizado. En este sentido, el terapeuta debe intentar adaptarse lo mejor posible a esta situación, a veces conflictiva, de coincidencia de distintos intereses y objetivos.

\section{Enfoques psicoterapéuticos}

La elección de la modalidad psicoterapéutica se hará en función del diagnóstico y de los tratamientos previos que haya recibido el paciente. Así, por ejemplo, la terapia cognitivo conductual podría ser la mejor opción para tratar a un trastorno de ansiedad o un trastorno obsesivo compulsivo en un adolescente. Por su parte, la terapia conductual es efectiva en los niños con problemas de conducta, que debido a su edad, todavía no son capaces de implicarse en otras formas de psicoterapia. En ocasiones lo más apropiado es una terapia de familia; y otras veces, el abordaje psicopedagógico de una dislexia o disgrafia, que pueden generar ansiedad en el niño, es el tratamiento de elección (68).

Una vez elegido el tratamiento, los padres, y siempre que sea posible, el paciente, deben dar su consentimiento informado. El terapeuta tiene la obligación de explicar lo que le sucede al paciente, qué se basa la elección terapéutica, las ventajas/desventajas de aplicarla y de no hacerlo, otras alternativas de tratamiento y los recursos disponibles.

En la infancia y la adolescencia, las formas de psicoterapia con mayor nivel de evidencia son: 1. Terapia de apoyo; 2. Terapia de conducta; 3 . Terapia cognitivo-conductual; 4. Resolución de problemas y aprendizaje de habilidades sociales; 5 . Psicoeducación y entrenamiento de padres; 6.Terapia familiar; 7. Terapia dialéctica; 8. Resolución de contingencias en el ámbito escolar; y, 9. Tratamiento de los trastornos específicos del aprendizaje. A continuación se describirán brevemente tres de ellas.

\section{Terapia conductual}

La terapia conductual se basa en los principios de la teoría del aprendizaje y su objetivo es modificar el comportamiento. En población pediátrica, comenzó a aplicarse en niños con discapacidad intelectual, autismo, trastornos de ansiedad y problemas de conducta, constatándose la importancia no sólo de la conducta en sí misma, sino también de los pensamientos e ideas que le acompañan, lo que condujo luego al desarrollo de la terapia cognitivo conductual.

Las técnicas de la terapia conductual tienen como objetivo reforzar, desarrollar o mantener una conducta deseada y reducir o eliminar una conducta no deseada. La terapia conductual está indicada para tratar los trastornos de ansiedad, la depresión, los trastornos de conducta, 
el trastorno por déficit de atención e hiperactividad, el autismo, la enuresis, la encopresis y la manifestaciones conductuales de la discapacidad intelectual (70).

\section{Terapia cognitivo-conductual}

Como se mencionó precedentemente en el caso de los adultos, la terapia cognitivo conductual se centra en el rol que tienen las distorsiones cognitivas en la aparición, desarrollo y persistencia de los síntomas psicopatológicos. Tiene como objetivo normalizar la conducta y los sentimientos del paciente, cambiando su modo de percibir y juzgar la realidad y, por lo tanto, cambiando su manera de pensar. En el caso de pacientes pediátricos, los padres deben estar activamente involucrados: entendiendo las características de la terapia y corregir sus propias distorsiones cognitivas con respecto a lo que le sucede a su hijo.

Numerosos estudios han evidenciado que, en niños y adolescentes, la terapia cognitivo conductual es superior, tanto comparada con placebo como con otras formas de psicoterapia. Esto puede ser observado en la depresión, la ansiedad, el trastorno obsesivo compulsivo, el trastorno de conducta y el trastorno negativista desafiante, entre otros $(71,72)$.

\section{Terapia familiar}

Las intervenciones terapéuticas basadas en la terapia familiar contemplan al síntoma del individuo como la manifestación de una disfunción del sistema de relación familiar. Centran su foco en el grupo completo aun cuando hubiera un trastorno mental diagnosticable y diferenciado en uno sus miembros. La terapia familiar insiste en reconocer la existencia de disfunciones familiares encubiertas que interactúan con las disfunciones explícitas de uno o más de sus integrantes, dificultando el tratamiento y ensombreciendo resultados.

\section{PSICOTERAPIA EN ADULTOS MAYORES}

La psicoterapia puede ser utilizada de manera muy eficaz en el tratamiento de los adultos mayores, ya sea como terapéutica primaria o adyuvante. Los principios básicos de los tratamientos psicoterapéuticos son similares a los de las personas más jóvenes; no obstante, deben tenerse en cuenta algunas temáticas propias de este grupo etario a las que darle atención (7376). La tabla 8 resume estas temáticas.

TABLA 8. TEMÁTICAS PROPIAS DEL ADULTO MAYOR EN PSICOTERAPIA

\begin{tabular}{|c|l|}
\hline Autoestima y autoconcepto & $\begin{array}{l}\text { La autoestima es a menudo un tema importante durante la psicoterapia con } \\
\text { pacientes de edad avanzada. El trabajo del terapeuta es entender cómo el } \\
\text { paciente intenta mantener la autoestima, a pesar de sus pérdidas biopsico- } \\
\text { sociales asociadas al envejecimiento, y apoyar o ampliar sus estrategias de } \\
\text { afrontamiento. Generalmente, el paciente adulto mayor busca aprobación y } \\
\text { validación de su terapeuta. El beneficio de esta validación es que ayuda a res- } \\
\text { taurar una visión positiva de sí mismo. }\end{array}$ \\
\hline Enfrentar las pérdidas & $\begin{array}{l}\text { Una temática recurrente en la terapia con pacientes adultos mayores es en- } \\
\text { frentar las pérdidas. Inevitablemente a lo largo de los años, y particularmente } \\
\text { a la edad avanzada, una persona pudo haber perdido a su pareja, amigos, } \\
\text { trabajo, roles, funciones e independencia, que a su vez hace que se generen } \\
\text { miedos y angustia anticipada hacia perdidas futuras. El terapeuta debe ser }\end{array}$ \\
\hline
\end{tabular}


capaz de ayudar al paciente a enfrentar las pérdidas de manera funcional y adaptativa.

Para pacientes ancianos enfrentando los muchos cambios del envejecimiento, incluyendo la posible dependencia física y la enfermedad, la psicote-

Enfrentar al envejecimiento, la enfermedad y la posible dependencia rapia puede ofrecer una relación segura y confiable a través de la cual examinar sus sentimientos de miedo. Estos sentimientos, incluyen, ser "odiado" o "maltratado" por su cuidador o no resistir la idea de depender completamente de alguien. La psicoterapia puede ayudar a crear o reforzar algunos recursos internos para hacer frente a estos procesos.

En la vejez, la muerte inminente se convierte en una realidad que ya no se puede negar, y enfrentar esto puede ser un verdadero desafío. Para una persona de edad con una "función del yo" pobre, esta tarea puede ser muy abru-

Enfrentar la muerte madora. Sin embargo, la ansiedad relacionada con la muerte es más intensa en la vida adulta temprana que en la vejez, ya que para las personas de edad avanzada, el dolor y el sufrimiento relacionados con la muerte son más preocupados que la muerte misma. El terapeuta debe trabajar esos miedos, buscando generar estrategias de afrontamiento saludables en el paciente.

Existe un fenómeno de transferencia única en la psicoterapia con los ancianos, en la que el terapeuta es visto como el "hijo" del paciente, fenómeno llamado "transferencia reversa". Esta debe ser tenida en cuenta durante el tratamiento, a fin de que no dificulte el proceso terapéutico. Por su parte, el terapeuta deberá prestar especial atención a los sentimientos positivos o negativos que él tiene hacia el paciente. Para el terapeuta, una relación con una persona mayor puede provocar ansiedad a causa de los posibles problemas físicos y dependencia que la persona mayor pueda tener. Esta ansiedad del terapeuta, a su vez, puede promover el miedo al paciente y puede provocar dificultades terapéuticas. Asimismo, la sobreidentificación del terapeuta con los problemas del paciente puede decantarse en sentimientos de tristeza y excesiva compasión, que pueden bloquear la empatía precisa y la exploración realista de la problemática del paciente.

Por tanto, y finalizando, las temáticas descriptas en la tabla 8 deben guiar la intervención psicoterapéutica del adulto mayor, que debe ser adaptada, individualizada y flexible y orientada a los retos asociados con el envejecimiento.

\section{CONCLUSIÓN}

En las últimas décadas se ha asistido a un aumento considerable de la investigación en el campo de la psicoterapia, lo que se traduce en la posibilidad de contar con más y mejores herramientas terapéuticas para abordar de un modo integral y eficiente distintos tipos de trastornos.

Así, la psicoterapia ha ido ocupando un espacio cada vez más importante en tanto herramienta socialmente útil, transformándose en un recurso innegable en el campo de la salud mental, no sólo como medio (psicológico) dirigido al tratamiento de los trastornos mentales, al alivio del sufrimiento sintomatológico o los cambios comportamentales, sino que su campo de acción asistencial se ha extendido también a las enfermedades físicas o a los padecimientos sociales que se vinculan a perturbaciones psicológicas. Además, en forma combinada con otros tipos de tratamiento (como el tratamiento psicofarmacológico), y ya no limitada estric- 
tamente a la enfermedad, la psicoterapia es un procedimiento útil para promover el desarroIlo, afrontar las crisis vitales y favorecer cambios inherentes al crecimiento mental y a la afirmación de la salud, es decir en la prevención y promoción de la misma (1).

A partir de ahora, las psicoterapias deben continuar enfrentándose al reto de demostrar que son tratamientos eficaces, estableciéndose en qué trastornos están indicadas, cuáles son sus posibles efectos adversos, el tiempo y el modo de aplicación y los criterios de suspensión. Es decir, las psicoterapias deben continuar demostrando, a través de estudios clínicos rigurosos, que son eficaces con niños y adolescentes, adultos y adultos mayores (77).

Con base en lo anterior, se hace perentorio continuar en la senda de determinar, de manera precisa, criterios basados en la evidencia y en las particularidades de cada paciente, para la indicación de intervenciones psicoterapéuticas adecuadas. Esta tarea deberá ir necesariamente unida a la formación de los médicos y otros profesionales de la salud, no solo desde el inicio de sus carreras sino de manera continua.

\section{CONFLICTOS DE INTERÉS Y FUENTE DE FINANCIACIÓN}

Los autores declaran no poseer conflictos de interés. Fuente de financiación: ninguna.

\section{REFERENCIAS BIBLIOGRÁFICAS}

1. Psiquiatria.com [Internet]. Madrid: Psiquiatria.com; c2002 [citado 18 ene 2017]. Fundamentos de psicoterapia. Breve reseña histórica de sus distintos enfoques teóricos y clínicos [aprox. 5 pantallas]. Disponible en: https://www.psiquiatria.com/historia/fundamentos-de-psicoterapia-breve-resena-historica-de-sus-distintos-enfoques-teoricos-y-clinicos/

2. Bernardi R, Defey D, Garbarino A, Tutté J, Villalba L. Guía clínica para la psicoterapia. Rev Psiquiatr Urug 2004;68(2):99-146. URL.

3. Valdivieso Fernández S. Fundamentos y límites de la psicoterapia. Boletín Esc. de Medicina, P. Universidad Católica de Chile 1994;23:97-102. URL.

4. Muñoz San José A, López Gómez D, Rodríguez Vega B. Peregrín E, Albillo D. Psicoterapia I: definición, indicación y evaluación de casos. En: Bravo MF, Saiz J, Bobes J, editores. Manual del Residente en Psiquiatría. Tomo II. 1a ed. Madrid: ENE Life Publicidad; 2009. p. 561-568.

5. Poch J, Ávila Espada A. Investigación en Psicoterapia: La contribución psicoanalítica. 1ạ ed. Barcelona: Paidós; 1997.

6. American Psychological Association [sede Web]. Washington DC: American Psychological Association; c2012 [citado 19 de ene 2017]. Recognition of Psychotherapy Effectiveness [aprox. 5 pantallas]. Disponible en: http://www.apa.org/about/policy/resolution-psychotherapy.aspx

7. Norcross JC. An eclectic definition of psychotherapy. In: Zeig J, Munion W, eds. What is psychotherapy? Contemporary perspectives. San Francisco CA: Jossey-Bass; 1990. p. 218-220.

8. Norcross JC. Psychotherapy relationships that work: Evidence-based responsiveness. 2nd ed. New York: Oxford University Press; 2011.

9. Fernández Liria A, Rodríguez Vega B. La práctica de la Psicoterapia. La construcción de narrativas terapéuticas. 1aㅡ ed. Bilbao: Desclée de Brouwer; 2001. 
10. Fernández Álvarez H. Fundamentos de un modelo integrativo en psicoterapia. 1a ed. Buenos Aires: Paidós; 1992.

11. Mariñelarena-Dondena L. Psicología positiva y modelos integrativos en psicoterapia. Fundamentos en Humanidades 2008;2(18):55-69. URL.

12. Sadock BJ, Sadock VA, Ruiz P. Kaplan \& Sadock Sinopsis de Psiquiatría. 11a ed. Barcelona: Wolters Kluwer; 2015.

13. Kandel E. Biology and the future of psychoanalysis: a new intellectual framework for psychiatry revisited. Am J Psychiatry 1999;156(4):505-524. URL.

14. Gabbard GO, Kay J. The fate of integrated treatment: whatever happened to the biopsychosocial psychiatrist? Am J Psychiatry. 2001;158(12):1956-1963. https://doi.org/10.1176/appi.ajp.158.12.1956

15. Schore AN. Effects of a secure attachment relationship on right brain development, affect regulation, and infant mental health. Infant Mental Health J 2001; 22(1-2):7-66. https://doi.org/10.1002/1097-0355(200101/04)22:1<7::AID-IMHJ2>3.0.CO;2-N

16. Wampold BE. The basic of psychotherapy: An introduction to theory and practice. Washington DC: American Psychological Association Press; 2010.

17. Gabbard GO. Empirical evidence and psychotherapy: a growing scientific base. Am J Psychiatry 2001; 158:1-3. https://doi.org/10.1176/appi.ajp.158.1.1

18. Cebolla Lorenzo S, Román Mazuecos ME, Palao Tarrero A. Psicoterapia II: abordaje psicoterapéutico desde las diferentes perspectivas. En: Bravo MF, Saiz J, Bobes J, editores. Manual del Residente en Psiquiatría. Tomo II. 1a ed. Madrid: ENE Life Publicidad; 2009. p. 569-576.

19. Bernardi R. The need for true controversies in psychoanalysis: the debates on Melanie Klein and Jacques Lacan in the Rio de la Plata. Int J Psychoanal 2002;83(4):851-873. $\underline{U R L}$.

20. de Maat S, de Jonghe F, Schoevers R, Dekker J. The effectiveness of long-term psychoanalytic therapy: A systematic review of empirical studies. Harvard Review of Psychiatry 2009;17(1):1-23. https://doi.org/10.1080/10673220902742476

21. Hofmann SG, Asnaani A, Vonk I, Sawyer AT, Fang A. The Efficacy of Cognitive Behavioral Therapy: A Review of Meta-analyses. Cognit Ther Res. 2012; 36(5): 427-440. https://doi.org/10.1007/s10608-012-9476-1

22. Beck AT. Cognitive therapy: Nature and relation to behavior therapy. J Psychother Pract Res. 1993;2(4):342-356. URL.

23. Ellis A. Reason and emotion in psychotherapy. 1st ed. New York: Lyle Stuart; 1962.

24. Ellis A, Grieger R. Manual de terapia racional emotiva. 1a ed. Bilbao: DDB; 1981.

25. Feixas G, Miró MT. Aproximaciones a la psicoterapia. 1a ed. Barcelona: Editorial Paidós; 1993.

26. Sluzki C. Interacción familiar: aportes fundamentales sobre teoría y técnica. Buenos Aires: Tiempo contemporáneo; 1971.

27. Stratton P. The Evidence Base of Systemic Family and Couples Therapies. Washington DC: The Association for Family Therapy \& Systemic Practice; 2011.

28. Hayes SC. Acceptance and commitment therapy, relational frame theory, and the third wave of behavioral and cognitive therapies. Behavior Therapy 2004; 35: 639-665. https://doi.org/10.1016/S0005-7894(04)80013-3

29. Luciano Soriano MC, Valdivia Salas MS. La terapia de aceptación y compromiso (ACT). Fundamentos, características y evidencias. Papeles del Psicólogo 2006;27(2):79-91. $\underline{\text { URL. }}$ 
30. Gutiérrez O, Luciano MC, Rodríguez M, Fink B. Comparison between an acceptancebased and a cognitive-control-based protocol for coping with pain. Behavior Therapy 2004;35:767-783. https://doi.org/10.1016/S0005-7894(04)80019-4

31. Hayes SC, Masuda A, Bissett R, Luoma J, Guerrero LF. BDT, FAP, and ACT: How empirically oriented are the new behavior therapy technologies? Behavior Therapy 2004;35:35-54. https://doi.org/10.1016/S0005-7894(04)80003-0

32. Linehan MM. Cognitive-behavioral treatment of bordeline personality disorder. Nueva York: The Guilford Press; 1993.

33. Hayes SC, Strosahl KD, Wilson KG. Acceptance and commitment therapy. Nueva York: The Guilford Press; 1999.

34. Segal ZV, Williams JM, Teasdale JD. Mindfulness-based cognitive therapy for depression. A new approach to preventing relapse. Nueva York: The Guilford Press; 2002.

35. Arce Ramírez A, Torales Benítez J, Cuellar Hoppe M, Ortiz MB, Minck Benítez N. Trastornos de Ansiedad. En: Arce Ramírez A, Torales Benítez J, editores. El Libro Azul de la Psiquiatría. 1a ed. Asunción: EFACIM; 2012. p. 149-174.

36. Ramírez Henderson R, Vargas Madriz LF. Terapia conductual dialéctica: descripción general de una propuesta centrada en la aceptación incondicional. Rev. Ciencias Sociales 2012;137:53-64. URL.

37. Gempeler J. Terapia conductual dialéctica. Revista Colombiana de Psiquiatría 2008;37(1):136-148. URL.

38. Davenport J, Bore M, Campbell J. Changes in personality in pre- and post-dialectical behaviour therapy borderline personality disorder groups. A question of selfcontrol.
Australian
Psychologist
2010;45(1):59-66. https://doi.org/10.1080/00050060903280512

39. Kliem S, Kröger C, Kosfelder J. Dialectical behavior therapy for borderline personality disorder: a meta-analysis using mixed-effects modeling. Journal of Consulting and Clinical Psychology 2010;78(6):936-951. http://dx.doi.org/10.1037/a0021015

40. Feigenbaum J. Dialectical behaviour therapy: an increasing evidence base. Journal of Mental Health 2007;16(1):51-68. http://dx.doi.org/10.1080/09638230601182094

41. Swales M. Dialectical behaviour therapy: description, research and future directions. International Journal of Behavioral Consultation and Therapy 2009;5(2):164-177. URL.

42. Wilson K, Luciano C. Terapia de Aceptación y Compromiso (ACT): un tratamiento conductual orientado a los valores. Madrid: Ediciones Pirámide; 2002.

43. Páez M, Gutiérrez O, Valdivia S, Luciano C. Terapia de Aceptación y Compromiso (ACT) y la importancia de los valores personales en el contexto de la terapia psicológica. International Journal of Psychology and Psychological Therapy 2006;6(1):1-20. URL.

44. Ruiz FJ. A review of Acceptance and Commitment Therapy (ACT) empirical evidence: Correlational, experimental psychopathology, component and outcome studies. International Journal of Psychology and Psychological Therapy 2010;10:125-162. URL.

45. Yovel I. Acceptance and Commitment Therapy and the new generation of cognitive behavioral treatments. Isr J Psychiatry Relat Sci. 2009;46(4):304-309. URL.

46. Cebolla i Martí A, Miró Barrachina MT. Efectos de la Terapia Cognitiva basada en la Atención Plena: una aproximación cualitativa. Apuntes de Psicología 2008;26(2):257268. URL.

47. Garay CJ, Korman GP. El modelo de Terapia Cognitiva Basada en la Conciencia Plena (mindfulness). Revista Argentina de Clínica Psicológica 2012;21(1):5-13. URL. 
48. Grossman P, Niemann L, Schmidt S, Walach H. Mindfulness-based stress reduction and health benefits: a meta-analysis. Journal of Psychosomatic Research 2004;57:35-43. http://dx.doi.org/10.1016/S0022-3999(03)00573-7

49. Beck AT. The current state of cognitive therapy: a 40-year retrospective. Arch Gen Psychiatry. 2005;62(9):953-959. http://dx.doi.org/10.1001/archpsyc.62.9.953

50. Chorpita BF, Daleiden EL, Ebesutani C, Young J, Becker KD, Nakamura BJ, et al. Evidence-Based Treatments for Children and Adolescents: An Updated Review of Indicators of Efficacy and Effectiveness. Clinical Psychology: Science and Practice 2011;18:154-172. http://dx.doi.org/10.1111/j.1468-2850.2011.01247.x

51. Beutler LE. Making science matter in clinical practice: Redefining psychotherapy. Clinical Psychology: Science and Practice 2009;16:301-317. http://dx.doi.org/10.1111/j.1468-2850.2009.01168.x

52. Hollon S, Stewart MO, Strunk D. Enduring effects for cognitive behavior therapy in the treatment of depression and anxiety. Annual Review of Psychology 2006;57:285-315. http://dx.doi.org/10.1146/annurev.psych.57.102904.190044

53. Shedler J. The efficacy of psychodynamic psychotherapy. Am Psychol. 2010;65(2):98109. http://dx.doi.org/10.1037/a0018378

54. Thomas R, Zimmer-Gembeck MJ. Behavioural outcomes of Parent-Child Interaction Therapy and trip P-Positive Parenting Program: A review and meta-analysis. J Abnorm Child Psychol. 2007;35(3):475-495. http://dx.doi.org/10.1007/s10802-007-9104-9

55. McMain S, Pos AE. Advances in psychotherapy of personality disorders: A research update. Current Psychiatry Reports 2007;9:46-52. URL.

56. Fullana MA, Fernández de la Cruz L, Bulbena A, Toro J. Eficacia de la terapia cognitivoconductual para los trastornos mentales. Medicina Clínica 2012; 138(5):215-219. http://dx.doi.org/10.1016/i.medcli.2011.02.017

57. Song $F$, Huttunen-Lenz M, Holland R. Effectiveness of complex psycho-educational interventions for smoking relapse prevention: an exploratory meta-analysis. J Public Health (Oxf). 2010;32(3):350-539. http://dx.doi.org/10.1093/pubmed/fdp109

58. Zimmermann G, Favrod J, Trieu VH, Pomini V. The effect of cognitive behavioral treatment on the positive symptoms of schizophrenia spectrum disorders: a meta-analysis. Schizophr Res. 20051;77(1):1-9. http://dx.doi.org/10.1016/j.schres.2005.02.018

59. Tolin DF. Is cognitive-behavioral therapy more effective than other therapies? A metaanalytic review. Clin Psychol Rev. 2010;30(6):710-720. http://dx.doi.org/10.1016/i.cpr.2010.05.003

60. Hofmann SG, Smits JAJ. Cognitive-behavioral therapy for adult anxiety disorders: a meta-analysis of randomized placebo-controlled trials. J Clin Psychiatry. 2008;69(4):621-632. URL.

61. Torales Benítez J, Medina Pereira A, Valenzuela Hermosa C, Fernández De los Ríos P. Trastorno Obsesivo Compulsivo. En: Arce Ramírez A, Torales Benítez J, editores. El Libro Azul de la Psiquiatría. 1a ed. Asunción: EFACIM, 2012. p. 175-184.

62. Bleichhardt G, Timmer B, Rief W. Cognitive-behavioural therapy for patients with multiple somatoform symptoms--a randomised controlled trial in tertiary care. J Psychosom Res. 2004;56(4):449-454. http://dx.doi.org/10.1016/S0022-3999(03)00630-5

63. Torales J, Arce A, Zacarías M, Girala N, Moreno M, Szwako A, et al. La Guía TAZ de Psicofarmacología Clínica. 1a ed. Asunción: EFACIM; 2014.

64. Dautovich ND, McNamara J, Williams JM, Cross NJ, McCrae CS. Tackling sleeplessness: psychological treatment options for insomnia. Nat Sci Sleep. 2010;2:23-37. URL. 
65. Saini M. A meta-analysis of the psychological treatment of anger: developing guidelines for evidence based practice. J Am Acad Psychiatry Law. 2009;37(4):473-488. URL.

66. Dixon-Gordon K, Turner BJ, Chapman A. Psychotherapy for personality disorders. Int Rev Psychiatry. 2011;23(3):282-302. http://dx.doi.org/10.3109/09540261.2011.58699

67. Pedreira Massa JL. Psicoterapia breve en la consulta para problemas funcionales frecuentes. En: AEPap ed. Curso de Actualización Pediatría 2004. Madrid: Exlibris Ediciones; 2004. p. 279-296.

68. Mardomingo Sanz MJ, Herreros Rodríguez O. Psicoterapias en niños y adolescentes. En: Soutullo Esperón C, Mardomingo Sanz MJ, editores. Manual de Psiquiatría del niño y del adolescente. 1a ed. Madrid: Editorial Médica Panamericana; 2010. p. 367-380.

69. Universidad Internacional de Valencia [Internet]. Valencia: Viu.es; c2014 [citado 18 ene 2017]. Psicoterapia infantil: principales modelos en la actualidad [aprox. 5 pantallas]. Disponible en: http://www.viu.es/psicoterapia-infantil-principales-modelos-en-la-actualidad/

70. Flannery-Schroeder EC, Kendall PC. Group and individual cognitive-behavioral treatments for youth with anxiety disorders: a randomized clinical trial. Cogn Ther Res 2000;24:252-278. URL.

71. McCarty C, Weisz JR. Effects of psychotherapy for depression in children and adolescents: what we can (and can't) learn from meta-analysis and component profile. J Am Acad Child Adolesc Psychiatry. 2007;46(7):879-886. http://dx.doi.org/10.1097/chi.0b013e31805467b3

72. Storch EA, Greffken GR, Merlo IJ, Mann G, Duke D, Manson M, et al. Family-based cognitive-behavioral therapy for pediatric obsessive-compulsive disorder: comparison of intensive and weekly approaches. J Am Acad Child Adolesc Psychiatry. 2007;46(4):469478. URL.

73. Atiq R. Common Themes and Issues in Geriatric Psychotherapy. Psychiatry (Edgmont) 2006;3(6):53-56. URL.

74. Pollack G. On ageing and psychotherapy. Int J Psychoanal. 1982;63:275-281.

75. Settlage CF. Transcending old age: Creativity, development, and psychoanalysis in the life of a centenarian. Int J Psychoanal. 1996; 77(Pt 3):549-564. URL.

76. Areán PA, Cook BL. Psychotherapy and combined psychotherapy/pharmacotherapy for late life depression. Biol Psychiatry. 2002;52(3):293-303. URL.

77. Gómez B, Salgueiro MC. Terapia cognitiva y medicación: un dilema en la trinchera. Revista Argentina de Clínica Psicológica 2007;16(3):205-218. URL. 